\title{
Extracorporeal Polypectomy
}

Endoscopic polypectomy is the principal form of treatment for colonic polyps. However, some polyps - particularly large, broadstalked ones - present problems to the endoscopist. During snaring of a large-stalked polyp, the head of the polyp tends to flop about, inevitably touching the bowel wall in several places. Current may leak at each point of contact, resulting in potential contralateral burns (1). We present here a case in which a large, broad-stalked and prolapsing rectal polyp was resected outside the body using an endoscopic polypectomy technique.

A 78-year-old man was admitted to our department in January 1996 due to frequent bowel movements and the appearance of a prolapsing mass from the anus during defecation. The anal inspection was normal. On digital examination of the rectum, a large, soft mass, filling the lumen, was felt. Rectosigmoidal examination showed a large polyp measuring $8 \mathrm{~cm}$ in diameter and with a stalk $2 \mathrm{~cm}$ in diameter, $5 \mathrm{~cm}$ from the anus. Endoscopic polypectomy appeared impossible with the polyp in place. The head of the polyp was so large that it would not have been possible to grasp it with a polypectomy snare. Even if it could have been snared, there would be a severe risk of burning adjacent tissues by touching the bowel wall over a large area. To avoid the risk of burning, we planned to carry out the polypectomy while the polyp was prolapsing. The patient was asked to strain, and the polyp prolapsed from the anus (Figure 1). To avoid hemorrhage, the stalk of the polyp was sutured. The polyp was then covered with a latex surgical glove, and the sleeve of the glove was introduced into the rectum (Figure 2). The fingers of the glove were cut, and a polypectomy snare loop was placed over the polyp head. Stepwise resection was performed using an Olympus electrosurgical unit. The head of the polyp was resected completely, without any complications. The day after, the patient was checked, and the remaining stalk of the polyp was resected intrarectally using a routine endoscopic polypectomy method. Microscopic examination of the polyp showed that it was a villous adenoma, with carcinoma in situ at the apical region. Rectosigmoidoscopy was repeated one month later, and the polyp site was seen to have healed completely,

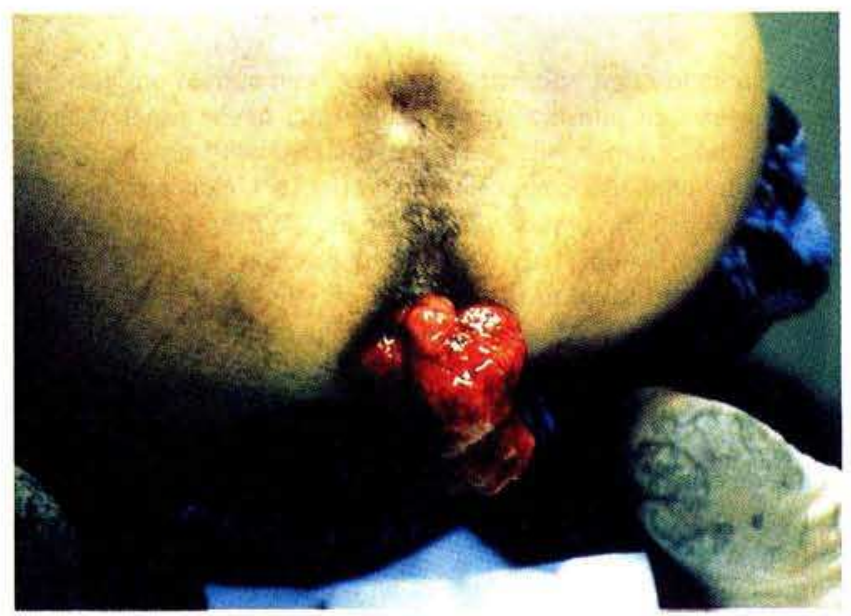

Figure 1: View of the prolapsing rectal polyp.

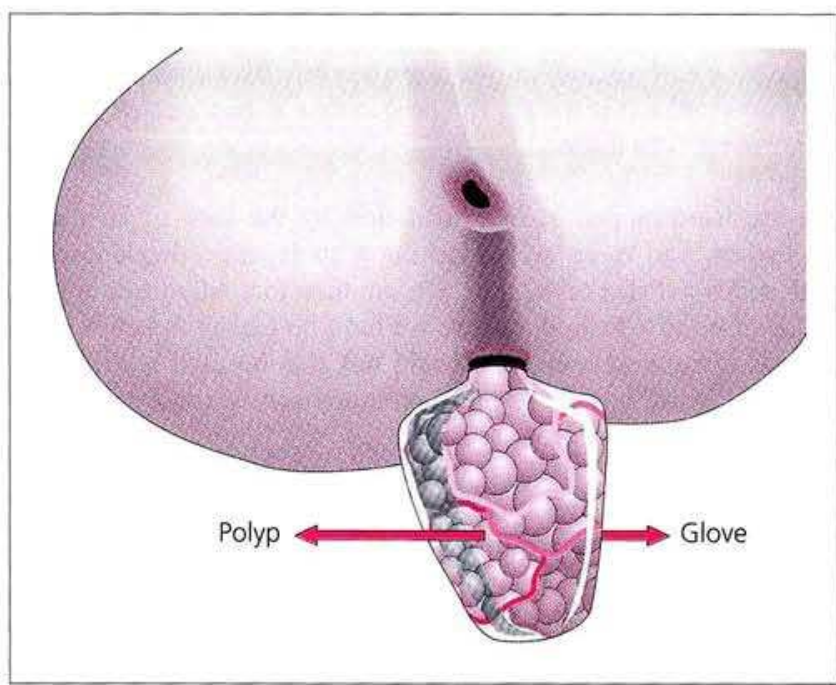

Figure 2: Covering the polyp with a latex glove before polypectomy. 
Large and stalked prolapsing rectal polyps can thus be resected safely outside the body using the method described.

A. Aydin, A. Musogłu, G. Ersöz, F. Günşar

Dept. of Gastroenterology, Medical Faculty, Ege University, Izmir, Turkey

\section{Reference}

1. Cotton PB, Williams CB. Practical gastrointestinal endoscopy. Oxford: Blackwell Scientific, 1990.
Corresponding Author

A. Aydin, M.D.

Dept. of Gastroenterology

Medical Faculty

Ege University

35100 Bornova-Izmir

Turkey

Fax: +90-232-3881969 